\title{
Identification of gastric atrophic changes: from histopathology to endoscopy
}

Authors

Institutions
Mário Dinis-Ribeiro ${ }^{1,2}$, Ernst J. Kuipers ${ }^{3}$

Department of Gastroenterology, Portuguese Institute of Oncology, Porto, Portugal

CIDES/CINTESIS, Faculty of Medicine, University of Porto, Porto, Portugal

Erasmus MC, Rotterdam, The Netherlands
Bibliography

DOI http://dx.doi.org/

$10.1055 / \mathrm{s}-0034-1392151$

Published online: 13.5.2015

Endoscopy 2015; 47: 533-537

(c) Georg Thieme Verlag KG

Stuttgart · New York

ISSN 0013-726X

\section{Corresponding author}

Mário Dinis-Ribeiro, MD

Department of

Gastroenterology

Portuguese Institute

of Oncology

Rua Dr. Bernardino de Almeida 4200-072 Porto

Portugal

Fax: +351225084055

mario@med.up.pt
In an aging European population, an increasing number of individuals will suffer from gastric cancer in the coming two decades. Recent research has determined the risk for gastric cancer in patients with different stages of gastric atrophy. Based on these data, it is now recommended that surveillance is offered to individuals with advanced stages of atrophic gastritis. Endoscopic biopsies of the gastric antrum and corpus are recommended in order to assess the severity and extent of gastric atrophy. This enables identification of those at highest risk of progressing to cancer. However, systematic reviews have shown that in recent years many researchers have assessed

\section{A challenge: multiple biopsies are required to determine the "premalignant stomach" \\ $\nabla$}

Nearly a quarter of human cancers arise in the gastrointestinal tract [1]. Interestingly, almost with no exception, these cancers are accessible by endoscopy and have identifiable and often treatable precursors. For example, Barrett's esophagus and colonic adenomas are well-known precursors of adenocarcinoma at their respective sites. Both lesions are widely studied, with a focus on improvement in their detection, natural history, and impact of endoscopic intervention [2, 3]. However, according to Western literature, the stomach seems to be the outlier in this discussion. This is surprising because the number of gastric cancer cases is expected to increase in Europe in the next two decades [1]. The lack of interest is likely due to a variety of reasons, including the prevailing misbelief that gastric cancer is a vanishing disease. Furthermore, endoscopic features of atrophic gastritis have been poorly defined. They also affect larger fields than Barrett's esophagus or colonic adenoma. However, these perspectives are changing. First, current image-enhanced endoscopy has greatly improved the accuracy of new endoscopic technologies for their accuracy in determining the severity and extent of gastric atrophy and metaplasia without the use of histology. Simple, reliable and accurate endoscopic features have been identified that can be used to either target biopsies or avoid biopsy sampling in the absence of endoscopic features of atrophy and intestinal metaplasia. This may largely simplify everyday practice. Randomized trials or large observational studies are now needed to demonstrate the accuracy of endoscopic assessment of the entire gastric mucosa and its impact on patient management.

endoscopic detection of atrophy and metaplasia [4]. Second, the risk of cancer in an affected individual appears distinctly higher than the average risk in patients with Barrett's esophagus or after removal of sporadic colonic adenomas [5-8]. There is therefore a need to reset the clinical and research agendas.

In the stomach, gland loss and intestinal metaplasia have long been recognized to be associated with an increased risk of gastric cancer. The first studies establishing this correlation were conducted as far back as the 1950s, and were originally based on fluoroscopy-guided gastric biopsies without endoscopy. This was in fact prior to the recognition of an association between Barrett's esophagus and colonic adenoma with adenocarcinoma at these sites. Further research on gastric atrophy was at times slow, but has increased in recent years. This has led to recent guidelines recommending that individuals with extensive, severe atrophy and metaplasia should be identified and kept under surveillance $[5,9]$.

These recommendations were based on studies that used random gastric biopsies to assess gastric atrophy and metaplasia, which can be multifocal. For this purpose, biopsies should be obtained from both the antrum and corpus. Several studies 


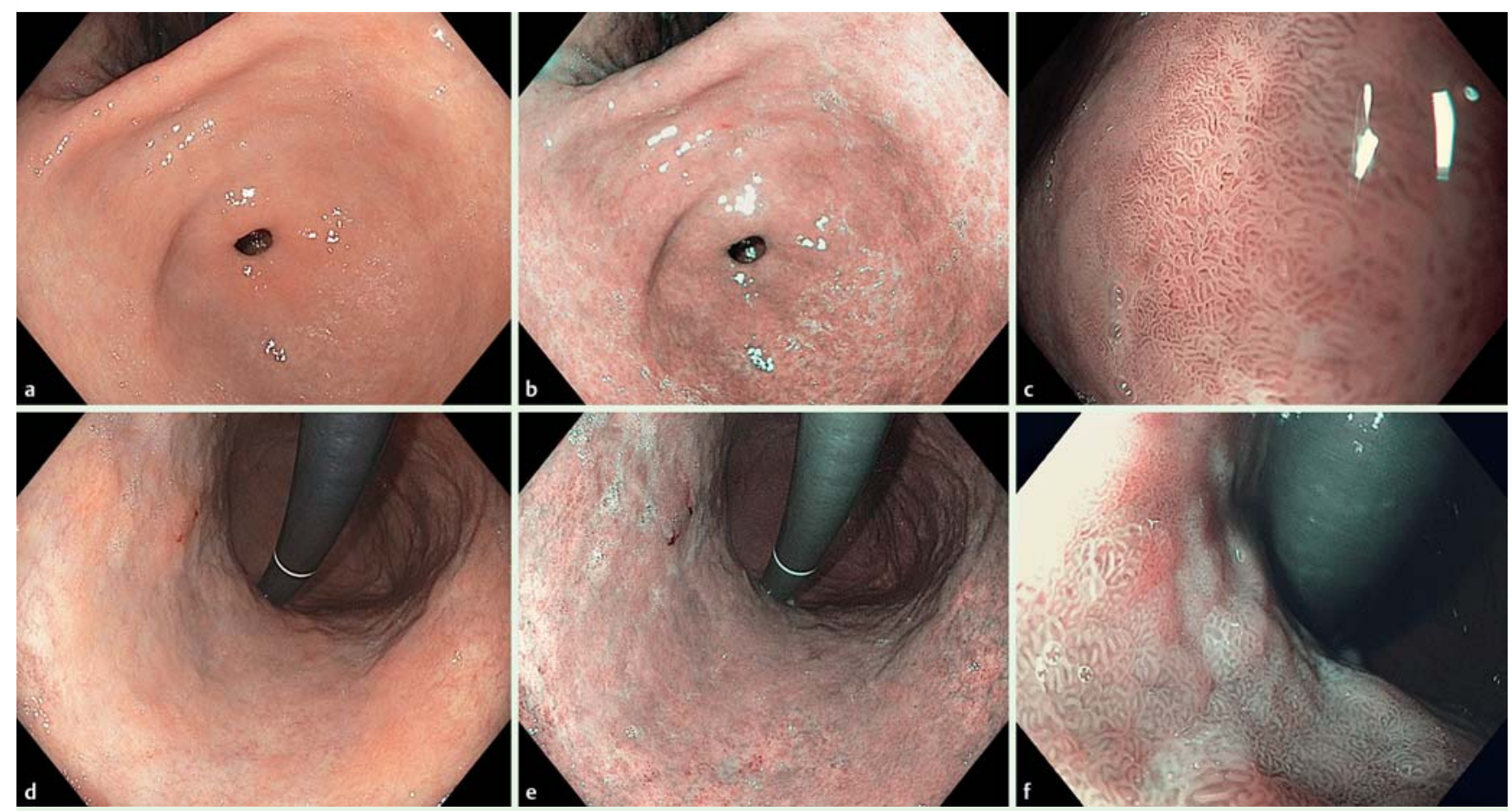

Fig. 1 Antrum (images $\mathbf{a}-\mathbf{c}$ ) and lesser curvature of the gastric body (images $\mathbf{d}-\mathbf{f}$ ), assessed with white-light endoscopy (a, d), and non-magnifying narrowband imaging at standard range $(\mathbf{b}, \mathbf{e})$, and close range $(\mathbf{c}, \mathbf{f})$. The antrum mucosa is normal. The lesser curvature shows a site with light blue crest, characteristic of intestinal metaplasia in the corpus (e).

have shown that diagnostic accuracy increases with the number of biopsies, but for practical reasons the numbers are usually limited to at least two from the antrum and two from the corpus. Efforts have been made to translate the resulting histological information into staging rules using either atrophy or intestinal metaplasia, which is a more advanced precancerous lesion and is associated with a higher observer reproducibility. These staging systems cross-tabulate with the severity of atrophy or metaplasia in the antrum and corpus $[10,11]$. Patients with moderate to severe lesions at both locations are categorized as OLGA (Operative Link on Gastritis Assessment) or OLGIM (Operative Link on Gastric Intestinal Metaplasia Assessment) stage III to IV. These individuals have a significant risk for progression to dysplastic or neoplastic changes during follow-up $[9,12]$. This phenotype corresponds to low levels of serum pepsinogens and pepsinogen ratios. This serum-marker categorization is used in Japan for population screening to identify individuals who require surveillance [13].

Random biopsy sampling from the antrum and corpus, subsequent staging, and the recommendation for surveillance of subgroups provide means to identify and manage patients at risk for gastric cancer. However, one should recognize that random biopsy sampling may miss relevant lesions. These changes are multifocal, and without endoscopic targeting focal changes may easily be under- or overdiagnosed. Furthermore, the sampling of biopsies poses a burden to the routine endoscopy setting, both in terms of workload and cost. Thus, the accuracy and impact of image-enhanced endoscopy for assessment of premalignant gastric changes has become relevant. This is fully in line with developments in other areas, such as Barrett's esophagus and colorectal polyps.

\section{Another perspective on current evidence: image-enhanced endoscopy allows accurate assessment of gastric intestinal metaplasia $\nabla$}

Recent studies have focused on the accuracy of diverse technologies versus histopathology. These have been summarized in a recent systematic review [14], but here the following examples by Pimentel-Nunes, Lim, and So and their colleagues are considered. Pimentel-Nunes et al. [4] assessed the interobserver agreement of endoscopic recognition of premalignant gastric lesions with narrow-band imaging (NBI). This was done in derivation and validation cohorts of patients. After reviewing the literature, the same investigators determined both the reliability and accuracy of a simplified classification of mucosal and vascular patterns compatible with premalignant changes of the gastric mucosa. The investigators aimed to establish an optimal, reliable classification for targeting areas with intestinal metaplasia and to recognize neoplastic changes. The features that form the basis for the classification can be easily learned [15]. Based on these features, the authors recognized three patterns that respectively reflected absence of intestinal metaplasia or dysplasia, presence of intestinal metaplasia, and presence of neoplastic changes. The absence of a tubulovillous mucosal pattern (the so-called pattern B) or "light-blue crest" changes virtually allows endoscopists to exclude the presence of intestinal metaplasia. The presence of either of these features is strongly predictive of intestinal metaplasia (likelihood ratio of 4.75 and 5.13, respectively). This is particularly relevant to settings in which the prevalence of intestinal metaplasia is higher than $20 \%$, such as in older patients, those with a family history of gastric cancer, and in regions of intermediate to high incidence of gastric adenocarcinoma [16]. Two limitations of the study were that it was based on videos rather 


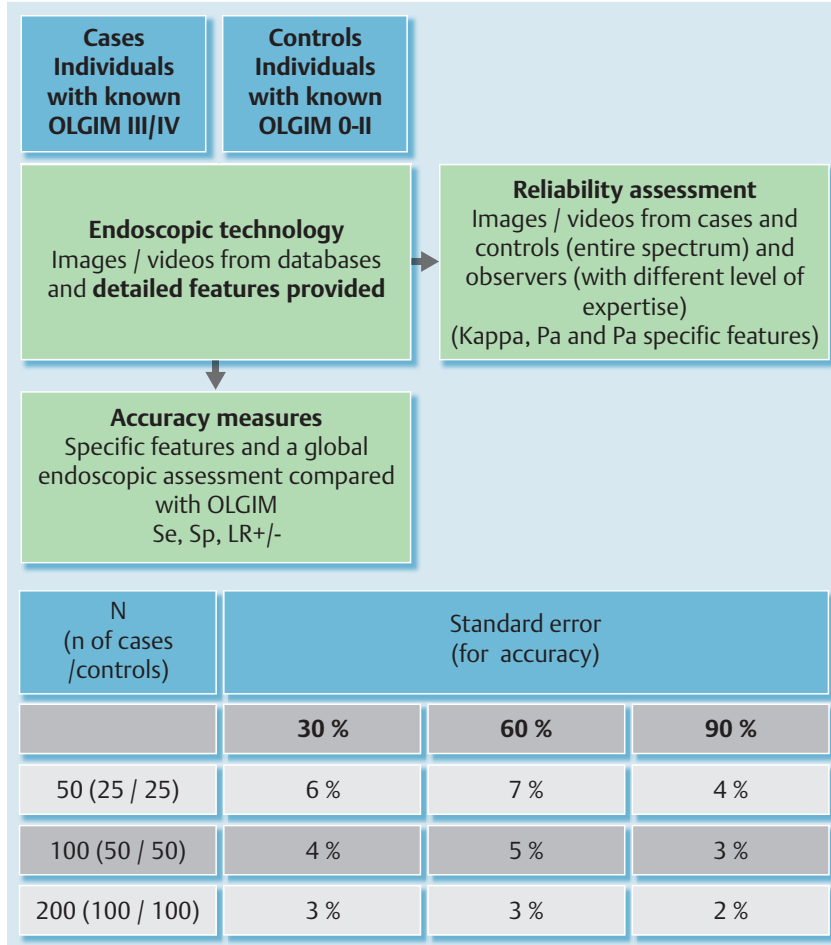

\begin{tabular}{|c|c|c|c|}
\hline \multicolumn{2}{|c|}{$\begin{array}{l}\text { Derivation cohort } \\
\text { Number depending on estimate } \\
\text { prevalence of OLGIM III/IV }\end{array}$} & & \\
\hline \multicolumn{2}{|c|}{$\begin{array}{l}\text { Endoscopic technology } \\
\text { Images / videos from databases and } \\
\text { detailed features provided }\end{array}$} & \multicolumn{2}{|c|}{$\begin{array}{c}\text { Reliability assessment } \\
\text { Images/videos from cases and } \\
\text { controls (entire spectrum) and } \\
\text { observers (with different level of } \\
\text { expertise) } \\
\text { (Kappa, Pa and Pa specific features) }\end{array}$} \\
\hline \multicolumn{4}{|c|}{$\downarrow$} \\
\hline \multicolumn{2}{|c|}{$\begin{array}{c}\text { Accuracy measures } \\
\text { Specific features and a global } \\
\text { endoscopic assessment compared } \\
\text { with OLGIM } \\
\text { Se, Sp, LR+/- }\end{array}$} & & \\
\hline \multicolumn{2}{|c|}{$\begin{array}{l}\text { Validation } \\
\text { Ideally multicenter }\end{array}$} & & \\
\hline \multicolumn{2}{|c|}{$\begin{array}{l}\text { Endoscopic technology } \\
\text { Images / videos from databases and } \\
\text { detailed features provided }\end{array}$} & & \\
\hline \multicolumn{2}{|c|}{$\downarrow$} & & \\
\hline \multicolumn{2}{|c|}{$\begin{array}{c}\text { Accuracy measures } \\
\text { Specific features and a global } \\
\text { endoscopic assessment compared } \\
\text { with OLGIM } \\
\text { Se, Sp, LR+/- }\end{array}$} & & \\
\hline \multirow[t]{2}{*}{$\mathrm{N}$} & \multicolumn{3}{|c|}{$\begin{array}{l}\text { Standard error } \\
\text { (for accuracy) }\end{array}$} \\
\hline & $30 \%$ & $60 \%$ & $90 \%$ \\
\hline 100 & $4 \%$ & $5 \%$ & $3 \%$ \\
\hline 200 & $3 \%$ & $3 \%$ & $2 \%$ \\
\hline 400 & $2 \%$ & $2 \%$ & $1 \%$ \\
\hline
\end{tabular}

Fig. 2 Suggested study designs. a Case-control studies can be used to identify endoscopic features, and to determine their reliability and accuracy for assessment of the OLGIM status of an individual patient. The table provides the number of patients to be included. Taped videos from individuals with known histology and status are assessed. The number of participants depends on the required accuracy estimates. Moreover, the use of likelihood ratios is suggested to overcome the dependency on prevalence of predictive values. Interobserver reliability assessment should be done by using sets of taped videos or images that incorporate the entire spectrum of "reality" (OLGIM 0 to IV). The different measures used include kappa, which may overcome chance agreement, and the proportion of agreement (i. e. that for specific features), which may allow improvement of descriptors. $\mathbf{b}$ Cohort studies can be conducted to derive and validate endoscopic features, and to determine accuracy in determining OLGIM status of interest. Retrospective (based on taped videos) or prospective cohorts may be designed where individual status is assessed based on endoscopic features (shown to be reliable) and accuracy measures have been estimated. A validation cohort, preferably in different centers, will ideally follow the derivation cohort. The number of patients to be included will depend on the desired standard error, but researchers should also consider expected prevalence of OLGIM III/IV (i.e. the a priori chance of finding these individuals). The table shows values based on a prevalence of advanced stages of approximately $20 \%$. se, sensitivity; sp, specificity; LR, likelihood ratio.

than life examinations, and no per-individual assessment was made.

In a second study, Lim et al. [17] assessed probe-based confocal endomicroscopy (pCLE) for the identification of intestinal metaplasia. This technology has already been used for the detection of dysplasia in other conditions (e.g. Barrett's esophagus, ulcerative colitis). The authors randomized 20 patients to conventional white light endoscopy (WLE) combined with either autofluorescence imaging (AFI) or NBI. All cases were also assessed using pCLE. Histology was then obtained for every site and was used as the gold standard. The study showed that pCLE adequately predicted the presence of intestinal metaplasia.
Finally, in a third study, So et al. [18] compared tri-modal imaging (WLE with AFI and magnified NBI) with WLE alone to accurately identify individuals with intestinal metaplasia. They used the same features as the Pimentel-Nunes study (pattern B and light blue crest). Again, the study showed that trimodal imaging adequately predicted the presence of intestinal metaplasia. In conclusion, several studies with different designs reported that image-enhanced endoscopy techniques allow endoscopists to reliably determine the presence of intestinal metaplasia ( $\bullet$ Fig. $\mathbf{1}$ ). This allows targeted biopsy sampling, with the advantage of reduced sampling and higher yield. This also addresses the multifocal nature of gastric atrophy and metaplasia. With use of these techniques, random biopsies can be replaced by targeted biopsies. 


\begin{tabular}{|c|c|c|c|}
\hline \multicolumn{4}{|c|}{ Patients } \\
\hline \multicolumn{2}{|c|}{ Endoscopic Technology A } & \multicolumn{2}{|c|}{ Endoscopic Technology B } \\
\hline \multicolumn{2}{|c|}{$\begin{array}{c}\text { Accuracy measures } \\
\text { Global endoscopic assessment } \\
\text { compared with OLGIM } \\
\text { Se, Sp, LR+/- }\end{array}$} & \multicolumn{2}{|c|}{$\begin{array}{c}\text { Accuracy measures } \\
\text { Global endoscopic assessment } \\
\text { compared with OLGIM } \\
\text { Se, Sp, LR+/- }\end{array}$} \\
\hline & \multicolumn{2}{|c|}{$\begin{array}{l}\text { Accuracy for } \\
\text { technology A }\end{array}$} & $\begin{array}{l}\text { Accuracy for } \\
\text { technology B }\end{array}$ \\
\hline & \multicolumn{2}{|c|}{$90 \%$} & $95 \%$ \\
\hline \multirow[t]{2}{*}{ N per group } & \multicolumn{2}{|c|}{432} & 432 \\
\hline & \multicolumn{2}{|c|}{$90 \%$} & $85 \%$ \\
\hline $\mathrm{N}$ per group & \multicolumn{2}{|c|}{197} & 197 \\
\hline
\end{tabular}

\begin{tabular}{|c|c|c|c|}
\hline \multicolumn{4}{|c|}{ Patients } \\
\hline \multicolumn{2}{|c|}{ Endoscopic Technology A } & \multicolumn{2}{|c|}{ Endoscopic Technology B } \\
\hline \multicolumn{2}{|c|}{ Endoscopic Technology B } & \multicolumn{2}{|c|}{ Endoscopic Technology A } \\
\hline \multicolumn{2}{|c|}{$\begin{array}{l}\text { Accuracy measures } \\
\text { Global endoscopic assessment } \\
\text { compared with OLGIM } \\
\text { Se, Sp, LR+/- }\end{array}$} & \multicolumn{2}{|c|}{$\begin{array}{c}\text { Accuracy measures } \\
\text { Global endoscopic assessment } \\
\text { compared with OLGIM } \\
\text { Se, Sp, LR+/- }\end{array}$} \\
\hline & \multicolumn{2}{|c|}{$\begin{array}{l}\text { Accuracy for } \\
\text { technology A }\end{array}$} & $\begin{array}{l}\text { Accuracy for } \\
\text { technology B }\end{array}$ \\
\hline & \multicolumn{2}{|c|}{$90 \%$} & $95 \%$ \\
\hline \multirow[t]{2}{*}{$\mathrm{N}$ per group } & \multicolumn{2}{|c|}{432} & 432 \\
\hline & \multicolumn{2}{|c|}{$90 \%$} & $85 \%$ \\
\hline $\mathrm{N}$ per group & \multicolumn{2}{|c|}{197} & 197 \\
\hline
\end{tabular}

Fig. 3 Suggestions for different randomized trials. a Patients can be randomized to receive different technologies. Note that the technology concept must consider endoscope characteristics (e. g. white-light endoscopy, narrow-band imaging, i-Scan), and detailed description of features (with previous assessment of their reliability), with histologic assessment (OLGIM) used as the gold standard. b Patients are randomized to receive different technologies in alternate sequence (crossover), and histologic assessment (OLGIM) is used as the gold standard. This design poses the need to consider that blinding must be adequately assured during gastroscopy, and presumes that biopsies are not made during first procedure (when researchers choose to do both interventions/technologies on the same day/time) or that time elapsed between gastroscopies is sufficient to overcome scarring from previous biopsies but short enough to avoid progression. The data in the tables are indicative suggestions, considering an $\alpha$ of $5 \%$ and power of $80 \%$. se, sensitivity; sp, specificity; LR, likelihood ratio.

\section{Take home messages}

$\nabla$

Research should focus on placing endoscopy as the gold standard for the premalignant stomach!

At this stage, it is important that further studies confirm and expand these findings. We hereby propose steps towards the generation of evidence to support or refute the use of currently available technologies in this setting. Briefly, the steps aim to move away from using histological examination of a specific site for a multifocal process, and to use staging systems as the gold standard to validate the use of endoscopy, and finally to adopt endoscopy as the new gold standard.

Studies should first focus on strengthening the evidence for use of the new image-enhanced endoscopy techniques for the assessment of intestinal changes in the gastric mucosa. These studies should also focus on intra- and interobserver consistency, and accuracy of severity grading. These aspects can first be addressed by use of video images of case studies. Histopathological assessment and classification of results using OLGIM can be used as the gold standard. Case-control studies ( $\bullet$ Fig.2a) or (preferably) validation cohorts $(\boldsymbol{O}$ Fig. $\mathbf{2 b}$ ) can further improve evidence.

Second, patients can be enrolled in studies with various designs: randomization to compare different technologies (different modalities of endoscopic assessment) ( $\mathbf{F i g . 3 a , b}$ ), with histology assessment as the outcome; or randomization to endoscopy vs. histology. In this case, other risk factors for individuals to harbor precancerous conditions should be recognized and assessed, as predictive values are affected by these.

Third, the evidence on the natural history of gastric precancerous lesions must be revisited in order to provide an accurate incidence rate for gastric cancer. This will help to optimize targeted surveillance, and also allow to refrain from surveillance in patients with a low risk phenotype. It will also help to schedule sur- veillance and further measures for those with premalignant stomachs.

Future studies should aim to identify individuals who are at risk, with sensitivities of $90 \%$ or above. In this assessment, a direct comparison should be made between endoscopic and histologic assessment using staging systems. Thus, both the presence and the distribution of premalignant lesions should be taken into account because both components translate into the risk for progression of disease. Endoscopic technologies should be considered in a continuum. Systems may well integrate non- and moderate-magnification techniques, with or without electronic chromoendoscopy (e.g. WLE, light-NBI) and high-magnification methods (e.g. pCLE). The final application of an endoscopic method to estimate an individual's risk of cancer will also depend on the epidemiological context such as the prevalence of Helicobacter pylori infection.

\section{Take home messages}

a) Current clinical guidelines suggest that random biopsies are required to assess the presence of multifocal gastric intestinal metaplasia - the phenotype carrying the highest risk for gastric adenocarcinoma.

b) Various studies and a systematic review suggest that image-enhanced endoscopy reliably and accurately determines the presence of intestinal metaplasia.

c) Further studies should confirm that targeted biopsies based on image-enhanced endoscopy can replace the current practice of routine random sampling.

d) Steps to build evidence for the use of gastric staging systems (i.e. OLGA, OLGIM) have been proposed. 


\section{Competing interests: None}

\section{References}

1 Ferlay J, Steliarova-Foucher E, Lortet-Tieulent J et al. Cancer incidence and mortality patterns in Europe: estimates for 40 countries in 2012. Eur J Cancer 2013; 49: 1374-1403

2 Anaparthy R, Sharma P. Progression of Barrett oesophagus: role of endoscopic and histological predictors. Nat Rev Gastroenterol Hepatol 2014; 11: 525-534

3 Kuipers EJ, Rösch T, Bretthauer M. Colorectal cancer screening - optimizing current strategies and new directions. Nat Rev Clin Oncol 2013; 10: 130-142

4 Pimentel-Nunes P, Dinis-Ribeiro $M$, Soares JB et al. A multicenter validation of an endoscopic classification with narrow band imaging for gastric precancerous and cancerous lesions. Endoscopy 2012; 44: 236 246

5 Dinis-Ribeiro M, Areia M, de Vries AC et al. Management of precancerous conditions and lesions in the stomach (MAPS): guideline from the European Society of Gastrointestinal Endoscopy (ESGE), European Helicobacter Study Group (EHSG), European Society of Pathology (ESP), and the Sociedade Portuguesa de Endoscopia Digestiva (SPED). Endoscopy 2012; 44: 74-94

6 de Vries AC, van Grieken NC, Looman CW et al. Gastric cancer risk in patients with premalignant gastric lesions: a nationwide cohort study in the Netherlands. Gastroenterology 2008; 134: 945-952

7 de Jonge PJ, van Blankenstein M, Looman CW et al. Risk of malignant progression in patients with Barrett's oesophagus: a Dutch nationwide cohort study. Gut 2010; 59: 1030-1036

8 Zauber AG, Winawer SJ, O'Brien MJ et al. Colonoscopic polypectomy and long-term prevention of colorectal-cancer deaths. N Engl J Med 2012; 366: $687-696$
9 den Hoed CM, Holster IL, Capelle LG et al. Follow-up of premalignant lesions in patients at risk for progression to gastric cancer. Endoscopy 2013; 45: 249-256

10 Rugge M, Meggio A, Pennelli $G$ et al. Gastritis staging in clinical practice: the OLGA staging system. Gut 2007; 56: 631-636

11 Capelle LG, de Vries AC, Haringsma J et al. The staging of gastritis with the OLGA system by using intestinal metaplasia as an accurate alternative for atrophic gastritis. Gastrointest Endosc 2010; 71: 1150-1158

12 Rugge M, de Boni M, Pennelli $G$ et al. Gastritis OLGA-staging and gastric cancer risk: a twelve-year clinico-pathological follow-up study. Aliment Pharmacol Ther 2010; 31: 1104-1111

13 Yoshida T, Kato J, Inoue I et al. Cancer development based on chronic active gastritis and resulting gastric atrophy as assessed by serum levels of pepsinogen and Helicobacter pylori antibody titer. Int J Cancer 2014; 134: 1445 - 1457

14 Kikuste I, Marques-Pereira R, Monteiro-Soares $M$ et al. Systematic review of the diagnosis of gastric premalignant conditions and neoplasia with high-resolution endoscopic technologies. Scand J Gastroenterol 2013; 48: 1108 - 1117

15 Dias-Silva D, Pimentel-Nunes $P$, Magalhães J et al. The learning curve for narrow-band imaging in the diagnosis of precancerous gastric lesions by using Web-based video. Gastrointest Endosc 2014; 79: 910-920

16 Marques-Silva L, Areia M, Elvas $L$ et al. Prevalence of gastric precancerous conditions: a systematic review and meta-analysis. Eur J Gastroenterol Hepatol 2014; 26: $378-387$

17 Lim LG, Yeoh KG, Srivastava S et al. Comparison of probe-based confocal endomicroscopy with virtual chromoendoscopy and white-light endoscopy for diagnosis of gastric intestinal metaplasia. Surg Endosc 2013; 27: 4649-4655

18 So J, Rajnakova A, Chan $\mathrm{Y}-\mathrm{H}$ et al. Endoscopic tri-modal imaging improves detection of gastric intestinal metaplasia among a high-risk patient population in Singapore. Dig Dis Sci 2013; 58: 3566 - 3575 